\title{
A model of Hall reconnection
}

\author{
Leonid M. Malyshkin* \\ Department of Astronomy \& Astrophysics, University of Chicago, 5640 S. Ellis Ave., Chicago, IL 60637
}

(Dated: October 24, 2018)

\begin{abstract}
The rate of quasi-stationary, two-dimensional magnetic reconnection is calculated in the framework of incompressible Hall magnetohydrodynamics (MHD), which includes the Hall and electron pressure terms in the Ohm's law. The Hall-MHD equations are solved in a local region across the reconnection electron layer, including only the upstream region and the layer center. In the case when the ion inertial length $d_{i}$ is larger than the Sweet-Parker reconnection layer thickness, the dimensionless reconnection rate is found to be independent of the electrical resistivity and equal to $d_{i} / L$, where $L$ is the scale length of the external magnetic field in the upstream region outside the electron layer, and the ion layer thickness is found to be $d_{i}$.

PACS numbers: 52.35.Vd, 94.30.cp, 96.60.Iv, 52.30.Ex
\end{abstract}

\section{INTRODUCTION}

Magnetic reconnection is a fundamental process of breaking and topological rearrangement of magnetic field lines in magnetized plasmas. Reconnection converts magnetic energy into kinetic and thermal energy and is believed to be responsible for many phenomena observed in the laboratory and cosmic plasmas [1, 2]. Because electrical resistivity is very low in hot plasmas, magnetic reconnection due to resistive dissipation of magnetic field is typically a very slow process [1, 2, 3]. Reconnection can become much faster in the case when resistivity is anomalously high due to local plasma instabilities [3, 4, 5]. Another possibility is fast reconnection made possible because of two-fluid plasma effects [1], which require a two-fluid magnetohydrodynamics (MHD) description of plasma. In the limit of zero electron-to-ion mass ratio, two-fluid MHD equations simplify and reduce to Hall-MHD equations. The later include the Hall and electron pressure terms in the Ohm's law, in addition to the resistivity term present in single-fluid MHD. Because of its relative simplicity, Hall-MHD description of plasma has been extensively used in numerical simulations of magnetic reconnection [6, 7, , 8, 9]. However, to the best of our knowledge, a simple self-consistent analytical model of Hall reconnection, similar to the classical Sweet-Parker model of resistive reconnection, has not been constructed. In this paper we consider HallMHD equations and present a theoretical model of Hall reconnection. Full two-fluid calculations for magnetic reconnection will be considered elsewhere. The analytical derivations of this paper are similar to the derivations done by Malyshkin, Linde \& Kulsrud [5] for the case of resistive single-fluid MHD reconnection.

\section{HALL-MHD EQUATIONS}

For simplicity and brevity, we use physical units in which the speed of light $c$ and four times $\pi$ are replaced by unity, $c=1$ and $4 \pi=1$. To rewrite our equations in the CGS units, one needs to make the following substitutions: magnetic field $\mathbf{B} \rightarrow \mathbf{B} / \sqrt{4 \pi}$, electric field $\mathbf{E} \rightarrow c \mathbf{E} / \sqrt{4 \pi}$, electric current $\mathbf{j} \rightarrow \sqrt{4 \pi} \mathbf{j} / c$, electrical resistivity $\eta \rightarrow$ $\eta c^{2} / 4 \pi$, and the proton electric charge $e \rightarrow \sqrt{4 \pi} e / c$.

We assume the plasma is non-relativistic, with both phase and physical velocities much smaller than the speed of light. We neglect electron inertia for the Hall-MHD description of plasma. The generalized Ohm's law is [10]

$$
\mathbf{E}=-\mathbf{V} \times \mathbf{B}+\eta \mathbf{j}+\frac{m_{i}}{\rho e} \mathbf{j} \times \mathbf{B}-\frac{m_{i}}{\rho e} \nabla \cdot P_{e},
$$

where $m_{i}$ is the ion mass, $\rho$ is the plasma density, $\mathbf{V}$ is the plasma velocity (equal to the ion velocity), $P_{e}$ is the tensor of the electron pressure. The first two terms on the right-hand side of Eq. (11) are the single-fluid MHD terms, the third and fourth terms are the Hall and electron pressure terms. The equation of plasma motion is [10]

$$
\rho \frac{\partial \mathbf{V}}{\partial t}+\rho(\mathbf{V} \cdot \nabla) \mathbf{V}=-\nabla \cdot P+\mathbf{j} \times \mathbf{B}
$$

where $P$ is the tensor of the total pressure (equal to the sum of the electron and ion pressure tensors), and we neglect plasma viscosity. Equation (2) appears exactly the same as in the case of single-fluid MHD. Note that $\nabla \cdot \mathbf{B}=0$, and, for non-relativistic plasma, $\nabla \cdot \mathbf{j}=0$.

We consider Hall magnetic reconnection in the classical two-dimensional Sweet-Parker-Petschek reconnection layer, shown in Fig. 11. The layer is in the $\mathrm{x}-\mathrm{y}$ plane with the $\mathrm{x}$ - and $\mathrm{y}$-axes being perpendicular to and along the layer respectively. All $\partial / \partial z$ derivatives are zero. The thickness of the reconnection layer is $2 \delta$, which is defined as the thickness of the out-of-plane current $\left(j_{z}\right)$ profile across the layer. Note that $2 \delta$ is approximately equal to the electron layer thickness, while the ion layer thickness $2 \Delta$ can be much larger. Velocity $V_{i n}$ is the plasma inflow velocity in the upstream region at point $M$, outside the electron layer. The magnetic field $B_{m}$ at point $M$ is in the y-direction. The out-of-plane field $B_{z}$ is assumed to have a quadrupole structure (see Fig.11), in agreement with nu- 


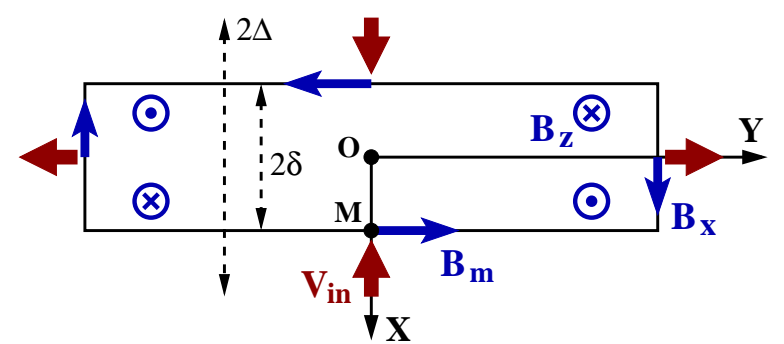

FIG. 1: Geometrical configuration of the reconnection layer.

merical simulations and laboratory experiments of twofluid reconnection 6, 7, 8, 9, 11, 12, 13, 14, 15, 16, 17 (a nearly uniform "guide" field component of $B_{z}$ is taken to be zero). The reconnection layer is assumed to have a point symmetry with respect to its geometric center point $O$ in Fig. 1 and reflection symmetries with respect to the axes $x$ and $y$. Thus, the $\mathrm{x}-, \mathrm{y}$ - and $\mathrm{z}$-components of $\mathbf{V}, \mathbf{B}$ and $\mathbf{j}$ have the following simple symmetries: $V_{x}( \pm x, \mp y)= \pm V_{x}(x, y), \quad V_{y}( \pm x, \mp y)=\mp V_{y}(x, y)$, $V_{z}( \pm x, \mp y)=V_{z}(x, y), \quad B_{x}( \pm x, \mp y)=\mp B_{x}(x, y)$, $B_{y}( \pm x, \mp y)= \pm B_{y}(x, y), \quad B_{z}( \pm x, \mp y)=-B_{z}(x, y)$, $j_{x}( \pm x, \mp y)= \pm j_{x}(x, y), j_{y}( \pm x, \mp y)=\mp j_{y}(x, y)$ and $j_{z}( \pm x, \mp y)=j_{z}(x, y)$. In the derivations presented below we will extensively use these symmetries.

\section{SOLUTION FOR HALL RECONNECTION}

Now let us list four assumptions that we make in this study. First, we assume that the plasma flow is incompressible inside the reconnection layer, $\rho=$ constant. Second, we assume that the electrical resistivity $\eta$ is constant and very small, so that the Lundquist number is very large. Third, we assume that the reconnection process is slow and quasi-stationary, so that we can neglect all time-derivatives $\partial / \partial t$ in Eqs. (11)-(2) and below. This assumption is satisfied if the reconnection rate is slow, $E_{z} \ll V_{A} B_{m}$, and there are no plasma instabilities in the reconnection layer. Fourth, we assume that the electron and ion pressure tensors are isotropic, so that the pressure tensors in Eqs. (1) and (2) are scalars.

Using Ampere's law and neglecting the displacement current, we find the $\mathrm{x}$ - and $\mathrm{y}$-components of the current to be $j_{x}=\partial_{y} B_{z}$ and $j_{y}=-\partial_{x} B_{z}$. Here and below we use convenient notations $\partial_{x} \equiv \partial / \partial x, \partial_{y} \equiv \partial / \partial y, \partial_{y y}^{2} \equiv$ $\partial^{2} / \partial y^{2}, \partial_{x y y}^{3} \equiv \partial^{3} / \partial x \partial y^{2}$, and etc. The z-component of the current at the reconnection layer central point $O$ is

$$
j_{o} \equiv\left(j_{z}\right)_{o}=\left(\partial_{x} B_{y}-\partial_{y} B_{x}\right)_{o} \approx B_{m} / \delta,
$$

where we use estimates $\left(\partial_{y} B_{x}\right)_{o} \ll\left(\partial_{x} B_{y}\right)_{o}$ and $\left(\partial_{x} B_{y}\right)_{o} \approx B_{m} / \delta$ at point $O$.

Next, neglecting the time derivative in Eq. (2), the equation for acceleration of plasma along the reconnection layer, in the y-direction, is $\rho(\mathbf{V} \cdot \nabla) V_{y}=-\partial_{y} P+$ $j_{z} B_{x}-j_{x} B_{z}$. We calculate the first order partial derivative $\partial / \partial y$ of this equation at point $\mathrm{O}$ and obtain

$$
\begin{aligned}
\rho\left(\partial_{y} V_{y}\right)_{o}^{2} & =-\left(\partial_{y y}^{2} P\right)_{o}+j_{o}\left(\partial_{y} B_{x}\right)_{o} \\
& \approx-B_{m}\left(\partial_{y y}^{2} B_{y}\right)_{m}+j_{o}\left(\partial_{y} B_{x}\right)_{o} \\
& =2 B_{m}^{2} / L^{2}+j_{o}\left(\partial_{y} B_{x}\right)_{o} .
\end{aligned}
$$

Here, we used the fact that the pressure term is $\left(\partial_{y y}^{2} P\right)_{o} \approx\left(\partial_{y y}^{2} B_{y}^{2} / 2\right)_{m}-\left(\partial_{y y}^{2} B_{z}^{2} / 2\right)_{o}=B_{m}\left(\partial_{y y}^{2} B_{y}\right)_{m}<$ 0 . Thus, the drop of pressure $P$ along the layer is equal to the magnetic pressure drop of the parallel field component outside the layer. This result follows from the force balance condition for the plasma across the reconnection layer (in analogy with the Sweet-Parker derivations for slowly inflowing plasma), and its rigorous proof can be found in [5]. The last expression in Eq. (4) is obtained by defining the magnetic field external scale as

$$
L^{2} \equiv-2 B_{m} /\left(\partial_{y y}^{2} B_{y}\right)_{m} .
$$

This is the scale of magnetic field just outside the reconnection electron layer (at point $\mathrm{M}$ ) and can be interpreted as the length of the layer. The $\left(\partial_{y} V_{y}\right)_{o}$ derivative on the left-hand-side of Eq. (4) can be estimated from plasma incompressibility condition at the O-point,

$$
\left(\partial_{y} V_{y}\right)_{o}=-\left(\partial_{x} V_{x}\right)_{o} \approx V_{i n} / \delta
$$

where we use an estimate $\left(\partial_{x} V_{x}\right)_{o} \approx-V_{\text {in }} / \delta$.

Next, the Faraday's law $\nabla \times \mathbf{E}=-\partial \mathbf{B} / \partial t$ for the $\mathbf{x}-$ and y-components of a quasi-stationary magnetic field in two dimensions is $\partial E_{z} / \partial y=-\partial B_{x} / \partial t \approx 0$ and $\partial E_{z} / \partial x=\partial B_{y} / \partial t \approx 0$. Therefore, the electric field zcomponent $E_{z}$ is constant in space, and from generalized Ohm's law (11) we obtain

$$
\begin{aligned}
\text { constant in space } \approx & E_{z}=-V_{x} B_{y}+V_{y} B_{x}+\eta j_{z} \\
& +\left(m_{p} / \rho e\right)\left(j_{x} B_{y}-j_{y} B_{x}\right) .
\end{aligned}
$$

Now, we use Eq. (7) to calculate $E_{z}$ at points $\mathrm{O}$ and M (see Fig. 11). At point $\mathrm{O}$ we have $E_{z}=\eta j_{o}$. At point $\mathrm{M}$ we have $E_{z}=V_{i n} B_{m}+\eta\left(j_{z}\right)_{m}+\left(m_{p} / \rho e\right) B_{m}\left(j_{x}\right)_{m} \approx$ $V_{i n} B_{m}+\eta\left(j_{z}\right)_{m}+\left(m_{p} / \rho e\right) B_{m}\left(\partial_{x y}^{2} B_{z}\right)_{o} \delta$, where we use an estimate $\left(j_{x}\right)_{m} \approx\left(\partial_{x} j_{x}\right)_{o} \delta=\left(\partial_{x y}^{2} B_{z}\right)_{o} \delta$. Equating these two expressions for $E_{z}$ at points $\mathrm{O}$ and $\mathrm{M}$ and neglecting the resistive term outside the reconnection layer at point $\mathrm{M}, \eta\left(j_{z}\right)_{m} \approx \eta B_{m} / L \ll \eta j_{o}$, we obtain

$$
\eta j_{o} \approx V_{i n} B_{m}+\left(m_{p} / \rho e\right) B_{m}\left(\partial_{x y}^{2} B_{z}\right)_{o} \delta .
$$

Next, we calculate the second order partial derivative $\partial^{2} / \partial y^{2}$ of Eq. (7) at point O. We have

$$
\begin{aligned}
0 \approx & 2\left(\partial_{y} V_{y}\right)_{o}\left(\partial_{y} B_{x}\right)_{o}+\eta\left(\partial_{y y}^{2} j_{z}\right)_{o} \\
& -2\left(m_{p} / \rho e\right)\left(\partial_{y} j_{y}\right)_{o}\left(\partial_{y} B_{x}\right)_{o} \\
\approx & 2\left(\partial_{y} V_{y}\right)_{o}\left(\partial_{y} B_{x}\right)_{o}-\eta\left(2 j_{o} / L^{2}\right) \\
& +2\left(m_{p} / \rho e\right)\left(\partial_{x y}^{2} B_{z}\right)_{o}\left(\partial_{y} B_{x}\right)_{o} .
\end{aligned}
$$


Here, to obtain the final expression, we use the fact that the y-scale of the current $j_{z}$, to a factor of order unity, is about the same as the $\mathrm{y}$-scale of the outside magnetic field, $j_{o}^{-1}\left(\partial_{y y}^{2} j_{z}\right)_{o} \approx B_{m}^{-1}\left(\partial_{y y}^{2} B_{y}\right)_{m}=-2 / L^{2}$. This result can be understood by taking the $\partial^{2} / \partial y^{2}$ partial derivative of the Ampere's law equation $j_{o} \approx B_{m} / \delta$, see Eq. (3), while keeping $\delta$ constant because the partial derivative in $y$ is to be taken at constant $x=\delta$ (for details see [5]).

Next, we use the z-component of the Faraday's law. We have $0 \approx-\partial B_{z} / \partial t=\partial_{x} E_{y}-\partial_{y} E_{x}$. We calculate the $\partial^{2} / \partial x \partial y$ partial derivative of this equation at point $\mathrm{O}$ and use Ohm's law (1) for $E_{x}$ and $E_{y}$. After tedious but straightforward derivations, we obtain

$$
\begin{aligned}
0 \approx & -\eta\left[\left(\partial_{x y x x}^{4} B_{z}\right)_{o}+\left(\partial_{x y y y}^{4} B_{z}\right)_{o}\right] \\
& -\left[\left(\partial_{y} B_{x}\right)_{o}\left(\partial_{x x}^{2} V_{z}\right)_{o}+\left(\partial_{x} B_{y}\right)_{o}\left(\partial_{y y}^{2} V_{z}\right)_{o}\right] \\
& +\left(m_{p} / \rho e\right)\left[\left(\partial_{y} B_{x}\right)_{o}\left(\partial_{x x}^{2} j_{z}\right)_{o}+\left(\partial_{x} B_{y}\right)_{o}\left(\partial_{y y}^{2} j_{z}\right)_{o}\right] \\
\approx & 2 \eta\left(\partial_{x y}^{2} B_{z}\right)_{o} / \delta^{2} \\
& -2\left(m_{p} / \rho e\right)\left[\left(j_{o} / \delta^{2}\right)\left(\partial_{y} B_{x}\right)_{o}+j_{o}^{2} / L^{2}\right]
\end{aligned}
$$

Here, to derive the final expression, we use $\left(\partial_{x y x x}^{4} B_{z}\right)_{o} \approx$ $-2\left(\partial_{x y}^{2} B_{z}\right)_{o} / \delta^{2},\left(\partial_{x y y y}^{4} B_{z}\right)_{o} \ll\left(\partial_{x y x x}^{4} B_{z}\right)_{o},\left(\partial_{x x}^{2} j_{z}\right)_{o} \approx$ $-2 j_{o} / \delta^{2},\left(\partial_{y y}^{2} j_{z}\right)_{o} \approx-2 j_{o} / L^{2}$ and $\left(\partial_{x} B_{y}\right)_{o} \approx j_{o}$. We also use formula $\left(\partial_{y} B_{x}\right)_{o}\left(\partial_{x x}^{2} V_{z}\right)_{o}+\left(\partial_{x} B_{y}\right)_{o}\left(\partial_{y y}^{2} V_{z}\right)_{o} \equiv$ 0 . To prove it, let us consider the z-component of plasma motion equation (2), which is $\rho V_{x}\left(\partial_{x} V_{z}\right)+$ $\rho V_{y}\left(\partial_{y} V_{z}\right)=j_{x} B_{y}-j_{y} B_{x}$. We calculate the $\partial^{2} / \partial x^{2}$ and $\partial^{2} / \partial y^{2}$ derivatives of this equation at point $\mathrm{O}$ and obtain $2 \rho\left(\partial_{x} V_{x}\right)_{o}\left(\partial_{x x}^{2} V_{z}\right)_{o}=2\left(\partial_{x} j_{x}\right)_{o}\left(\partial_{x} B_{y}\right)_{o}$ and $2 \rho\left(\partial_{y} V_{y}\right)_{o}\left(\partial_{y y}^{2} V_{z}\right)_{o}=-2\left(\partial_{y} j_{y}\right)_{o}\left(\partial_{y} B_{x}\right)_{o}$. Thus, we have $\left(\partial_{x x}^{2} V_{z}\right)_{o}=-\left(\partial_{x y}^{2} B_{z}\right)_{o}\left(\partial_{x} B_{y}\right)_{o} / \rho\left(\partial_{y} V_{y}\right)_{o}<0$, $\left(\partial_{y y}^{2} V_{z}\right)_{o}=\left(\partial_{x y}^{2} B_{z}\right)_{o}\left(\partial_{y} B_{x}\right)_{o} / \rho\left(\partial_{y} V_{y}\right)_{o}>0$ and $\left(\partial_{y} B_{x}\right)_{o}\left(\partial_{x x}^{2} V_{z}\right)_{o}+\left(\partial_{x} B_{y}\right)_{o}\left(\partial_{y y}^{2} V_{z}\right)_{o} \equiv 0$.

Now, we have six equations (3), (44), (6), (8), (9) and (10), and we have six unknowns: $j_{o}, \delta, V_{i n},\left(\partial_{y} V_{y}\right)_{o}$, $\left(\partial_{y} B_{x}\right)_{o}$ and $\left(\partial_{x y}^{2} B_{z}\right)_{o}$. Thus, we can solve for all unknown quantities. For convenience of presentation, we express the solution in terms of the Alfven velocity $V_{A}=$ $B_{m} / \sqrt{\rho}$, the ion inertial length $d_{i}=m_{i} / e \sqrt{\rho}$ and the Lundquist number $S=L V_{A} / \eta$. The solution is

$$
\begin{aligned}
j_{o} & \approx \frac{B_{m}}{L}\left(S \sqrt{3}+2 S^{2} d_{i}^{2} / L^{2}\right)^{1 / 2}, \\
\delta & \approx L\left(S \sqrt{3}+2 S^{2} d_{i}^{2} / L^{2}\right)^{-1 / 2}, \\
V_{i n} & \approx \sqrt{3} V_{A}\left(S \sqrt{3}+2 S^{2} d_{i}^{2} / L^{2}\right)^{-1 / 2}, \\
\left(\partial_{y} V_{y}\right)_{o} & \approx \sqrt{3} V_{A} / L \\
\left(\partial_{y} B_{x}\right)_{o} & \approx \frac{B_{m}}{L}\left(S \sqrt{3}+2 S^{2} d_{i}^{2} / L^{2}\right)^{-1 / 2}, \\
\left(\partial_{x y} B_{z}\right)_{o} & \approx 2 S d_{i} B_{m} / L^{3} \\
E_{z}=\eta j_{o} & \approx V_{A} B_{m}\left(S^{-1} \sqrt{3}+2 d_{i}^{2} / L^{2}\right)^{1 / 2}
\end{aligned}
$$

The last equation gives the reconnection rate $E_{z}$. In the limit $S \gg 1$ and $d_{i} \ll L$ the reconnection rate is slow, $E_{z} \ll V_{A} B_{m}$, and our assumption of a quasistationary reconnection process is self-consistent. Equation (14) implies that the ions are accelerated up to approximately Alfven velocity $V_{A}$ along the reconnection layer of length $L$. At the same time, the rate of electron acceleration along the layer at point $\mathrm{O}$ is $\left(\partial_{y} V_{y}^{e}\right)_{o}=\left(\partial_{y} V_{y}\right)_{o}-\left(m_{i} / \rho e\right)\left(\partial_{y} j_{y}\right)_{o}=\left(\partial_{y} V_{y}\right)_{o}+$ $\left(V_{A} d_{i} / B_{m}\right)\left(\partial_{x y} B_{z}\right)_{o} \approx\left(V_{A} / L\right)\left(\sqrt{3}+2 S d_{i}^{2} / L^{2}\right)$, where $\mathbf{V}^{e}$ denotes the electron velocity.

The ion layer thickness $2 \Delta$ can be estimated as follows. In the upstream region outside the ion layer at $x=\Delta$ ideal single-fluid MHD applies. Therefore, at $x=\Delta$ and $y=0$ the resistive and Hall terms in Eq. (7) can be neglected and $E_{z} \approx V_{R} B_{m}$, where $V_{R}$ is the plasma inflow velocity outside the ion layer. Velocity $V_{R} \approx E_{z} / B_{m}$ is called the reconnection velocity. It can also be estimated as $V_{R} \approx\left(\partial_{y} V_{y}\right)_{o} \Delta$. Thus,

$$
\Delta \approx \frac{E_{z}}{\left(\partial_{y} V_{y}\right)_{o} B_{m}} \approx \frac{L}{\sqrt{3}}\left(S^{-1} \sqrt{3}+2 d_{i}^{2} / L^{2}\right)^{1 / 2} .
$$

We discuss the solution (11)-(18) in the next section. Now, let us make an important remark. Our analytical derivations involve an approximate solution of the Hall-MHD equations in the infinitesimal neighborhood of line OM across the reconnection electron layer (see Fig. 1). All physical quantities, $j_{o}, \delta, V_{i n},\left(\partial_{y} V_{y}\right)_{o}$, $\left(\partial_{y} B_{x}\right)_{o},\left(\partial_{x y} B_{z}\right)_{o}, B_{m}$ and $L$, are defined either at point $\mathrm{O}$ (the layer center) or at point $\mathrm{M}$ (the upstream region). In other words, all our derivations involve only the upstream region and the layer center, and we do not need to consider the downstream region for estimation of the reconnection rate. This "local" equations approach was first developed in [5] for single-fluid MHD reconnection with anomalous electrical resistivity, and this approach works for Hall-MHD reconnection as well. Note however, that we define the field scale $L$ by Eq. (5), and its exact value, as well as the value of field $B_{m}$ in the upstream region, depend on the "global" solution of the Hall-MHD equations outside the reconnection layer. Both $L$ and $B_{m}$ enter our model as parameters. Determination of their values requires numerical simulations of the global field configuration and is not considered here.

\section{DISCUSSION}

When $d_{i} \ll L / \sqrt{S}=\delta_{\mathrm{SP}}\left(\delta_{\mathrm{SP}}\right.$ is the Sweet-Parker layer thickness), the solution (11)-(18) reduces to the SweetParker solution: $j_{o} \approx \sqrt{S} B_{m} / L, \delta \approx \Delta \approx L / \sqrt{S}=\delta_{\mathrm{SP}}$, $V_{i n} \approx V_{A} / \sqrt{S},\left(\partial_{y} V_{y}\right)_{o} \approx V_{A} / L,\left(\partial_{y} B_{x}\right)_{o} \approx B_{m} / L \sqrt{S}$, $E_{z} \approx V_{A} B_{m} / \sqrt{S}$ and $\left(\partial_{y} V_{y}^{e}\right)_{o} \approx\left(\partial_{y} V_{y}\right)_{o} \approx V_{A} / L$.

In the opposite limit, when $d_{i} \gg L / \sqrt{S}=\delta_{\mathrm{SP}}$ and 
reconnection is in a collisionless Hall regime, we have

$$
\begin{aligned}
j_{o} & \approx S d_{i} B_{m} / L^{2} \gg \sqrt{S} B_{m} / L, \\
\delta & \approx L^{2} / S d_{i} \ll L / \sqrt{S}=\delta_{\mathrm{SP}}, \\
\Delta & \approx d_{i} \gg L / \sqrt{S}=\delta_{\mathrm{SP}}, \\
V_{i n} & \approx V_{A} L / S d_{i} \ll V_{A} / \sqrt{S}, \\
\left(\partial_{y} V_{y}\right)_{o} & \approx V_{A} / L \\
\left(\partial_{y} B_{x}\right)_{o} & \approx B_{m} / S d_{i}, \\
\left(\partial_{x y} B_{z}\right)_{o} & \approx 2 S d_{i} B_{m} / L^{3}, \\
E_{z} & \approx\left(d_{i} / L\right) V_{A} B_{m} \gg V_{A} B_{m} / \sqrt{S} .
\end{aligned}
$$

The electron acceleration rate along the reconnection layer at O-point is $\left(\partial_{y} V_{y}^{e}\right)_{o} \approx S d_{i}^{2} V_{A} / L^{3} \gg$ $\left(\partial_{y} V_{y}\right)_{o}$. Fast electron outflow along the layer creates the quadrupole field $B_{z}$ [18]. From Eq. (26) we find that the rate of collisionless Hall reconnection, $E_{z}=$ $\left(d_{i} / L\right) V_{A} B_{m}$, is independent of the electrical resistivity $\eta$ [19]. The reconnection velocity is $V_{R} \approx E_{z} / B_{m} \approx$ $\left(d_{i} / L\right) V_{A}$. From Eq. (21) we find $\Delta \approx d_{i}$ for the ion layer thickness, which is in agreement with experiment [13].

In it noteworthy that in the absence of collisions, when $\eta \rightarrow 0$, it is an anisotropic electron pressure that balances $E_{z}$ field at point $O$. In this case electrons are accelerated by $E_{z}$ field during time $\sim L / V_{e T}$, while they are unmagnetized and are traveling with thermal speed $V_{e T}$ inside the electron layer of length $L[\underline{3}, 20]$. As a result, the effective resistivity becomes $\eta_{\text {eff }} \approx d_{e}^{2} V_{e T} / L$, where $d_{e}=\sqrt{m_{i} m_{e}} / e \sqrt{\rho}$ is the electron inertial length $\left(m_{e}\right.$ is the electron mass). Thus, pressure anisotropy and electron inertia become important (they will be considered elsewhere).

In the end of this paper, let us briefly discuss possible mechanisms of fast reconnection, which is independent of the macroscopic size of the reconnecting system. First, note that the collisionless Hall reconnection rate $E_{z}=\left(d_{i} / L\right) V_{A} B_{m}$ is high when the reconnection electron layer length $L$ is microscopically small and comparable to $d_{i}$. Although the value of $L$ cannot be determined in our model, numerical simulations find that $L$ can indeed be much smaller than the macroscopic system size [7, 8, 14, 15, 16, 17]. For example, $L$ can be as small as $\approx 10 d_{i}$ during quasi-stationary collisionless reconnection, resulting in a very fast reconnection rate $E_{z} \approx 0.1 V_{A} B_{m}[8,16,17$.

Another possible mechanism of fast reconnection can result from the dependence of the reconnection rate on the density $\rho$ and temperature $T$ of the plasma. We have $\eta \propto T^{-3 / 2}$ [10], $V_{A} \propto \rho^{-1 / 2}, S \propto \rho^{-1 / 2} T^{3 / 2}$, $d_{i}^{2} \propto \rho^{-1}$. Therefore, $d_{i} / \delta_{\mathrm{SP}} \propto(T / \rho)^{3 / 4}$, and the collisionless Hall reconnection rate is $E_{z} \propto \rho^{-1}$. Thus, even if initially $L \gg d_{i}$, as reconnection proceeds, the plasma is heated up by Joule heating, the plasma temperature rises, the plasma density drops, and the reconnection becomes more and more collisionless and faster and faster.
This run-away reconnection process can possibly operate in solar corona and Earth's magnetosphere. The dependence $E_{z} \propto \rho^{-1}$ also supports a model of self-regulation heating in solar corona [21].

I am especially grateful to Russell Kulsrud for many very helpful discussions and for pointing out to me the importance of electron pressure anisotropy. I would also like to thank Ellen Zweibel, Fausto Cattaneo, Masaaki Yamada, Boon Chye (BC) Low, Eugene Parker, Alex Obabko, Dmitri Uzdensky, Hantao Ji, Viacheslav Titov, Amitava Bhattacharjee and the anonymous referee for their interest in this work, discussions and for useful comments. This work was supported by the NSF Center for Magnetic Self-Organization (CMSO) in Laboratory and Astrophysical Plasmas at the University of Chicago.

A note added after publication: After the publication of his paper, the author has became aware that Simakov and Chacon (2008) have independently obtained a Hall reconnection rate formula, which is the same as Eq. (17) up to numerical factors of order unity [22]. Although the final result is basically the same, the two derivations are significantly different. The author has used a rigorous local analysis, in which the Hall-MHD equations are solved only in the upstream region and in the center of the reconnection layer. By contrast, Simakov and Chacon (2008) have used a different approach that also considers the downstream region. In particular, they have assumed that the thickness of the ion layer is given by $\max \left\{d_{i}, \delta\right\}$, and obtained their reconnection rate formula as an approximate interpolation between the rates in the collisional and collisionless regimes. There are also minor differences in the problem formulation; that of Simakov and Chacon (2008) includes electron viscosity, which is neglected by the author, and neglects electron pressure, which is included by the author. Expression $\left(d_{i} / L\right) V_{A}$ for the reconnection velocity in the collisionless regime was obtained earlier by Stanley W. H. Cowley (1985) [19].

* Electronic address: leonmal@uchicago.edu

[1] D. Biskamp, Magnetic Reconnection in Plasmas (Cambridge University Press, England, 2000).

[2] R. M. Kulsrud, Plasma Physics for Astrophysics (Princeton University Press, 2005).

[3] M. Yamada, R. Kulsrud and H. Ji, upcoming in Rev. Mod. Phys. (2009).

[4] R. M. Kulsrud, Earth, Planets and Space 53, 417 (2001); arXiv:astro-ph/0007075

[5] L. M. Malyshkin, T. Linde and R. M. Kulsrud, Physics of Plasmas 12, 102902 (2005).

[6] M. Hesse, J. Birn and M. Kuznetsova, J. Geophys. Res., 106, 3721 (2001).

[7] R. Fitzpatrick, Phys. Plasmas, 11, 937 (2004).

[8] J. D. Huba and L. I. Rudakov, Phys. Rev. Lett., 93, 175003 (2004). 
[9] N. A. Murphy and C. R. Sovinec, Phys. Plasmas, 15, 042313 (2008).

[10] P. A. Sturrock, Plasma Physics (Cambridge University Press, Cambridge, England, 1994).

[11] P. Ricci, J. U. Brackbill, W. Daughton and G. Lapenta, Phys. Plasmas, 11, 4102 (2004).

[12] A. Ishizawa, R. Horiuchi and H. Ohtani, Phys. Plasmas 11, 3579 (2004).

[13] M. Yamada, Y. Ren, H. Ji, J. Breslau, S. Gerhardt, R. Kulsrud and A. Kuritsyn, Phys. Plasmas 13, 052119 (2006).

[14] M. E. Mandt, R. E. Denton and J. F. Drake, Geophys. Res. Lett. 21, 73 (1994).

[15] A. Bhattacharjee, Z. W. Ma and X. Wang, Phys. Plasmas 8, 1829 (2001).
[16] M. A. Shay, J. F. Drake, B. N. Rogers and R. E. Denton, Geophys. Res. Lett. 26, 2163 (1999).

[17] M. A. Shay, J. F. Drake, M. Swisdak and B. N. Rogers, Phys. Plasmas, 11, 2199 (2004).

[18] D. A. Uzdensky and R. M. Kulsrud, Phys. Plasmas, 13, 062305 (2006).

[19] S. W. H. Cowley, in Solar System Magnetic Fields, edited by E. R. Priest (D. Reidel Publishing Company, 1985), 121.

[20] R. Kulsrud, H. Ji, W. Fox and M. Yamada, Phys. Plasmas 12, 082301 (2005).

[21] D. A. Uzdensky, Phys. Rev. Lett. 99, 261101 (2007).

[22] A. N. Simakov and L. Chacon, Phys. Rev. Lett. 101, 105003 (2008). 